\title{
ASSESSMENT OF ASYMMETRY IN BILATERAL STATIC FRONTAL BREAST THERMOGRAMS USING DIFFERENCE IMAGE AND RADIOMIC FEATURES
}

\author{
Vijaya Madhavi M. ${ }^{1}$ and T. Christy Bobby ${ }^{2}$ \\ ${ }^{1}$ Department of Electronics and Communication Engineering, East Point College of Engineering and \\ Technology, Bengaluru, Affiliated to Visvesvaraya Technological University, Belagavi, Karnataka, India \\ ${ }^{2}$ Department of Electronics and Communication Engineering, \\ M. S. Ramaiah University of Applied Sciences, Bengaluru, Karnataka, India
}

Corresponding author: Vijaya Madhavi M.

Email: vijayamadhavi79@gmail.com

https://doi.org/10.34107/BiomedSciInstrum.57.04256

\begin{abstract}
Asymmetry analysis of bilateral thermogram images is an important preliminary approach for breast cancer detection. The purpose of this work is to develop an automated algorithm to detect and classify symmetric and asymmetric bilateral static frontal breast thermograms $(\mathrm{N}=63)$. The images are pre-processed using anisotropic diffusion filter for removal of noise. Further, segmentation of complete breast region is carried out using level set segmentation without re-initialization. The bifurcation point is computed from the intersection point of interior inframammary curves attained using polynomial curve fitting on the boundary pixels. The obtained breast region is sliced vertically along this bifurcation point to obtain right and left breast sections. Image subtraction is performed between right breast image and flipped left breast image to obtain the difference image. The obtained difference image is sharpened and 144 texture features such as first-order statistical, cooccurrence, run length and laws energy features are extracted and Absolute Difference (AD) between symmetric and asymmetric subjects for each feature is computed. The features for which the value of AD is greater than 0.1 is considered as substantial features. Twenty four substantial features are obtained and are given as an input to Least Square Support Vector Machine (LSSVM) to automate the classification. The results shows that the maximum segmentation overlap measure obtained is $98.3 \%$. The classification accuracy obtained using LSSVM with Radial Basis Function (RBF) is 95.65\% and sensitivity, specificity and Area Under the Curve (AUC) are 100\%, 90.9\% and 0.9545 respectively. Thus the proposed methodology appears to be effective in detecting asymmetric heat patterns and hence can be deployed in thermal screening systems.
\end{abstract}

Keywords: bilateral frontal thermogram, anisotropic diffusion, radiomics, asymmetric heat pattern, LSSVM

\section{INTRODUCTION}

Breast cancer is a leading cancer type identified among women and is the second largest contributor of cancer death. In the year 2020, globocan 2020 estimates 2.2 million new cases and 0.68 million demises worldwide due to breast cancer [1]. The survival rate can be improved if the abnormal growth of cells is detected at its initial stage of occurrence. Thermogram is an evolving imaging technique that uses InfaRed (IR) radiations emitted from the body surface for the diagnosis of cancer. It has the ability to detect breast cancer before any other modality can detect it, thus making it preferable over other modalities [2].

Thermography is a non-invasive, contactless, radiation-free technique that employs a specialized IR camera to image the breast region. Cancerous and pre-cancerous tissues have high metabolic activity compared to normal tissues. Thus, these tissues require more nutrients and blood supply for its reckless growth resulting in increased regional temperature that can be captured by an IR camera. Hence a 